\title{
Mucormycosis presenting as orbital cellulitis; a case report
}

\begin{abstract}
A 68 year old man with myelodysplastic syndrome and transfusion related iron overload presented with orbital cellulitis found to be due to mucormycosis infection. He was treated with anti-fungals and extensive surgical debridement of the effected tissue. The aim of this article is to discuss mucormycosis and review the literature related to its diagnosis and management.
\end{abstract}

Keywords: mucormycosis, orbital cellulitis
Volume 3 Issue I - 2015

Stuart AJ, Lloyd GW

St George s Hospital, London

Correspondence: Stuart AJ, St George's Hospital, Tooting

London SE5 9RS, London, Email alastairstuart@nhs.net

Received: October 22, 2015 | Published: October 27, 2015
Abbreviations: RAPD, relative afferent pupillary defect; CT, computed tomography; FESS, functional endoscopic sinus surgery; ENT, ear nose \& throat; NPL, nil perception of light; CNS, central nervous system

\section{Introduction}

Mucormycosis is a rare invasive fungal infection caused by fungi from the Mucorales order. ${ }^{1}$ Mucormycosis is classified according to anatomical site of infection, ${ }^{2}$ with rhino-orbital-cerebral is the most common. ${ }^{3}$ This fungal infection begins from the sinonasal mucosa after inhalation of fungal spores and the aggressive and rapid progression of the disease may lead to orbital and brain involvement. ${ }^{4}$ Predisposing factors for mucormycosis include diabetes mellitus, ${ }^{3}$ systemic immunosuppression, ${ }^{5}$ (especially in patients with haematological malignancies, ${ }^{6}$ ) and iron overload. ${ }^{7}$ Mortality rates associated with mucormycosis vary depending on the site of infection and the initial health of the patient but have been reported to range between 40 and $70 \%{ }^{8}$

\section{Case presentation}

A 68 year old man presented to eye casualty at a district general hospital with a two day history of right-sided periorbital swelling. He had an existing diagnosis of myelodysplasia causing a refractory anaemia (excess blast type 2 cells) and was being treated with deferoxamine for transfusion related iron overload. Clinical examination revealed peri-orbital swelling and erythema. There was significant chemosis but a full range of eye movements and minimal proptosis. Vision was 6/9 and there was no RAPD or sign of optic nerve dysfunction. He was commenced or oral antibiotics but on review 24hours later his condition had worsened. A CT scan showed significant proptosis of the right globe and opacification of the anterior and posterior ethmoidal paranasal sinuses (Figure 1).

He was subsequently admitted for intravenous antibiotics (coamoxiclav). On day 2 of his admission his condition deteriorated rapidly and he developed rigors. Further CT imaging showed gas in the soft tissues around the globe and subsequently a right FESS and orbital decompression with samples for histology and microbiology was performed by the ENT team. His antibiotics were changed to Tazocin (Piperacillin/tazobactam), metronidazole and teicoplanin. On day 4 of his admission his vision in the right eye dropped suddenly to NPL secondary to central retinal artery occlusion. The samples from the FESS confirmed a diagnosis of mucormycosis (Lichthemia corymbifera) and he was commenced on Liposomal Amphotericin. He was transferred to a tertiary hospital for right orbital exenteration, external ethmoidectomy, medial maxillectomy, and resection of middle turbinate (Figure 2). On day 25 of his admission he underwent cosmetic repair of the right exenteration using skin harvested from the back. Over the coming months the patient required treatment for renal impairment and was treated continuously with systemic anti fungal medications (Posaconazole/Amphotericin). 8 months post admission his condition was stable with no sign of a recurrence of mucormycosis.

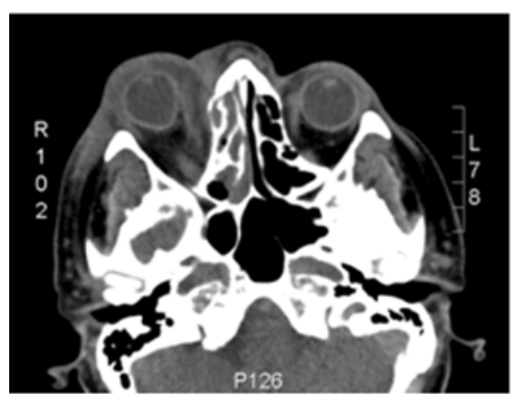

Figure I CT showing right globe proptosis and significant sinus disease.

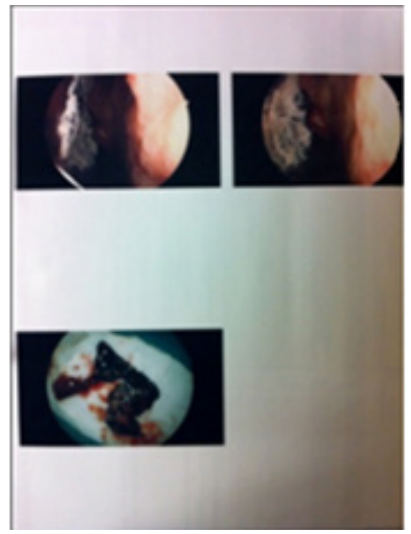

Figure 2 Images from nasal surgery showing black necrotic tissue and hyphae consistent with fungal infection. 


\section{Discussion}

Mucormycosis is a rare but devastating condition due to its rapid and aggressive angio-invasive growth. Orbital infection with mucormycosis is associated with a high risk of mortality as once the fungus enters the orbit it can easily access the intracranial space through the ophthalmic artery, optic canal or superior orbital fissure.

Risk factors for mucormycosis include diabetes mellitus, immune compromise, and iron overload. Diabetics are predisposed to the infection because an environment of low oxygen tension, hyperglycaemia and ketosis provides an excellent medium for the fungus to thrive. In a 20year retrospective case series of invasive fungal disease in the orbit and sinuses, ${ }^{9}$ all of the patients with mucormycosis were immune suppressed or had diabetes. Our patient was immunocompromised due to myelodysplastic syndrome. His treatment with frequent blood transfusions had also caused him to become overloaded with iron, further increasing his predisposition for mucormycosis infection. Iron is required by virtually all microbial pathogens for virulence and growth and sequestration of iron is a major host defence mechanism. ${ }^{10}$ It could therefore be assumed that treatment with iron chelators which reduce the amount of free serum iron would reduce the risk of infection by mucormycosis. However, it has been shown that patients treated with the iron chelator deferoxamine have a markedly increased incidence of invasive Mucormycosis. ${ }^{11}$ Although in the human host deferoxamine acts as iron chelator, mucormycosis fungi actually use it as a siderophore to access previously unavailable iron. ${ }^{12}$ It can accumulate 8 - to- 40 -foldgreater amounts of iron supplied by deferoxamine than Aspergillus fumigatus and Candida albicans, respectively, and this increased iron uptake by mucormycosis is linearly correlated with its growth in serum. ${ }^{13}$ Some research has shown certain iron chelators such as desafirox to be protective against mucormycosis in a mice model. ${ }^{14}$ However, in a human model desafirox was associated with higher incidence of mortality than placebo. ${ }^{15}$

Four factors are critical for eradicating mucormycosis: rapidity of diagnosis; reversal of the underlying predisposing factors (if possible); appropriate surgical debridement of infected tissue; and appropriate antifungal therapy. ${ }^{16}$ Early diagnosis is important because small, focal lesions can often be surgically excised before they progress to involve critical structures or disseminate. ${ }^{17}$ Correcting or controlling the risk factors previously discussed is essential to improve outcomes. Diabetic control should be optimized, whilst iron chelating and immunosuppressive therapies should be stopped. Tissue diagnosis can be very helpful and biopsies should be performed as soon as possible particularly as the appearance of tissue at endoscopy may lag behind invasion, as the mucosa can appear pink and viable during the initial phase of fungal invasion. ${ }^{17}$

The paucity of clinical trials regarding mucormycosis makes management of cases challenging for clinicians. The most established anti fungal for the treatment of mucormycosis is Amphotericin which has been used for over 50 years. ${ }^{16}$ Liposomal Amphotericin B (LAmB) has been shown to be less toxic than Amphotericin B and has improved CNS penetration. ${ }^{18}$ Itraconazole, ${ }^{19}$ and Posaconazole,${ }^{20}$ have been shown in case reports to work successfully and may be used as an adjunctive therapy.

Surgery is an essential part of the management of cases due to the massive amount of tissue necrosis occurring during mucormycosis, which may not be prevented by killing the organism. ${ }^{21}$ Surgical debridement of infective and necrotic tissue must be performed as a matter of urgency. In rhinocerebral mucormycosis, early surgical excision of the infected sinuses and appropriate debridement of the retro-orbital space can often prevent the infection from extending into the eye, thereby obviating the need for enucleation and resulting in extremely high cure rates $(>85 \%) .{ }^{17}$

\section{Conclusion}

Mucormycosis is a rare but life threatening fungal infection. Clinicians must have a high index of suspicion in rapidly progressive cases of orbital cellulitis and in cases with predisposing factors such as diabetes mellitus, immuno suppression and iron overload. The successful management of such cases centres around prompt diagnosis, appropriate anti-fungal agents and urgent surgical debridement of involved tissues.

\section{Acknowledgments}

None.

\section{Conflicts of interest}

The author declares that there are no conflicts of interest.

\section{Funding}

None.

\section{References}

1. James D William, Timothy Berger, Dirk Elston. Andrews' Diseases of the Skin: clinical Dermatology. USA: Saunders Elsevier. 2006;328.

2. Mohamed MS, Abdel-Motaleb HY, Mobarak FA. Management of rhinoorbital mucormycosis. Saudi Med. 2015;36(7):865-868.

3. Pinto ME, Manrique HA, Guevara X, et al. Hyperglycemic hyperosmolar state and rhino-orbital mucormycosis. Diabetes Res Clin Pract. 2011;91(2):e37-39.

4. Viterbo S, Fasolis M, Garzino-Demo P, et al. Management and outcomes of three cases of rhino-cerebral mucormycosis. Oral Surg Oral Med Oral Pathol Oral Radiol Endod. 2011;112(6):69-74.

5. Wali U, Balkhair A, Al-Mujaini A. Cerebro-rhino orbital mucormycosis: an update. J Infect Public Health. 2012;5(2):116-126.

6. Binder U, Maurer E, Lass-Flörl C. Mucormycosis--from the pathogens to the disease. Clin Microbiol Infect. 2014;20(6): 60-66.

7. Ibrahim AS. Host cell invasion in mucormycosis: role of iron. Curr Opin Microbiol. 2011;14(4):406-411.

8. Chamilos G, Lewis RE, Kontoyiannis DP. Delaying amphotericin B-based frontline therapy significantly increases mortality among patients with hematologic malignancy who have zygomycosis. Clin Infect Dis. 2008;47(4): 503-509.

9. D Trief, S Gray, F Jakobiec, et al. Br J Ophthalmol. 2015.

10. Collins H. Withholding iron as a cellular defence mechanism--friend or foe? Eur J Immunol. 2008;38(7):1803-1806.

11. Boelaert JR, Van Cutsem J, de Locht M, et al. Deferoxamine augments growth and pathogenicity of Rhizopus, while hydroxypyridinone chelators have no effect. Kidney Int. 1994;45(3):667-671. 
12. de Locht M, Boelaert JR, Schneider YJ. Iron uptake from ferrioxamine and from ferrirhizoferrin by germinating spores of Rhizopus microsporus. Biochem Pharmacol. 1994;47(10):1843-1850.

13. Boelaert JR, de Locht M, Van Cutsem J, et al. Mucormycosis during deferoxamine therapy is a siderophore-mediated infection. In vitro and in vivo animal studies. J Clin Invest. 1993;91(5):1979-1986.

14. Ibrahim AS, Gebermariam T, Fu Y, et al. The iron chelator deferasirox protects mice from mucormycosis through iron starvation. J Clin Invest. 2007;117(9): 2649-2657.

15. Spellberg B, Ibrahim AS, Chin-Hong PV, et al. The DeferasiroxAmBisome Therapy for Mucormycosis (DEFEAT Mucor) study: a randomized, double-blinded, placebo-controlled trial. J Antimicrob Chemother. 2012;67(3):715-722.

16. Spellberg B, Edwards J, Ibrahim A. Novel Perspectives on Mucormycosis: Pathophysiology, Presentation, and Management. Clin Microbiol Rev. 2005;18(3):556-569.
17. Nithyanandam S, Jacob MS, Battu RR, et al. Rhino-orbito-cerebral mucormycosis. A retrospective analysis of clinical features and treatment outcomes. Indian J Ophthalmol. 2003;51(3):231-236.

18. Groll AH, Giri N, Petraitis V, et al. Comparative efficacy and distribution of lipid formulations of amphotericin B in experimental Candida albicans infection of the central nervous system. J Infect Dis. 2000;182(1):274282.

19. Eisen DP, Robson J. Complete resolution of pulmonary Rhizopus oryzae infection with itraconazole treatment: more evidence of the utility of azoles for zygomycosis. Mycoses. 2004;47(3-4):159-162.

20. Ide L, Buysschaert I, Demuynck H, et al. Zygomycosis in neutropenic patients with past Aspergillus infection: a role for posaconazole?. Clin Microbiol Infect. 2004;10(9): 862-863.

21. Ibrahim AS, Spellberg B, Avanessian V, et al. Rhizopus oryzae adheres to, is phagocytosed by, and damages endothelial cells in vitro. Infect Immun 2005;73(2):778-783. 